\title{
A Note on the Fuzzy Linear Maps
}

\author{
Chang Bum Kim
}

Department of Mathematics, Kookmin University, Seoul 136-702, South Korea.

\begin{abstract}
In this paper we investigate some situations in connection with two exact sequences of fuzzy linear maps. Also we obtain a generalization of the work [Theorem 4] of Pan [5], and we study the analogies of The Four Lemma and The Five Lemma of homological algebra. Finally we obtain a special exact sequence.
\end{abstract}

Key Words: Fuzzy $R$-module, Fuzzy linear map(Fuzzy $R$-map), Fuzzy weak isomorphism, Quasi-monic, epic.

\section{Introduction}

Fuzzy modules were introduced by Negoita and Ralescu [1]. Katsaras and Liu [2], and Lowen [3] have developed the theory of fuzzy vector spaces.

Fu-Zhen Pan [4] investigated fuzzy vector spaces for the following purposes; to establish a fundamental frame of fuzzy vector space by virtue of homological algebra and modular theory, and to stretch it out to study general fuzzy modules.

In fact, fuzzy vector spaces are the simplest kind of fuzzy free modules. The theory of fuzzy modules has been a virgin field for a long time.

Recently, many authors presented the same research on fuzzy modules, properties of fuzzy finitely generated modules and fuzzy quotient modules, etc.

In particular, Fu-Zhen Pan [5] and Kim [6] investigated the properties of the sequence of fuzzy linear maps and studied the situations in connection with two exact sequences of fuzzy linear maps.

In this paper, we investigate some situations in connection with two exact sequences of fuzzy linear maps.

\section{Preliminaries}

In this section, we review some definitions and some results which will be used in the later sections. Throughout

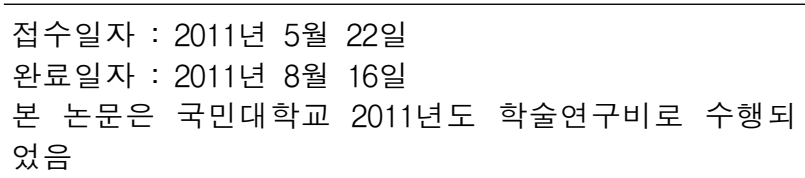

this paper, we assume that all modules are equipped with the same underlying commutative ring $R$.

Definition 2.1 ([5]). A $R$-module $M$ together with a function $\chi$ from $M$ into $[0,1]$ is called a fuzzy $R$-module if it satisfies the following conditions
(1) $\chi(a+b) \geq \min \{\chi(a), \chi(b)\}$
(2) $\chi(-a)=\chi(a)$
(3) $\chi(0)=1$
(4) $\chi(r a) \geq \chi(a)$,

for any $a, b \in M$ and $r \in R$, denoted it by $(M, \chi)$ or $\chi_{M}$.

Definition 2.2 ([5]). Let $\chi_{M}, \eta_{N}$ be any two fuzzy $R$ modules, then $\tilde{f}: \chi_{M} \longrightarrow \eta_{N}$ is called a fuzzy linear map (or fuzzy $R$-map) if there exists a linear map $f: M \longrightarrow N$ such that $\eta(f(a)) \geq \chi(a)$ for all $a \in M$.

Remark 2.3. Let $\chi_{M}, \eta_{N}$ be any two fuzzy $R$-modules. Then $\tilde{f}: \chi_{M} \longrightarrow \eta_{N}$ is called a fuzzy strong linear map if there exists a linear map $f: M \longrightarrow N$ such that $\eta(f(a))=$ $\chi(a)$ for all $a \in M$.

Definition 2.4 ([5]). Let $\tilde{f}: \chi_{M} \longrightarrow \eta_{N}$ be a fuzzy linear map. $\tilde{f}$ is called fuzzy weak isomorphism, denoted $\chi_{M} \simeq_{W}^{f} \eta_{N}$.

Definition 2.5 ([5]). For a fuzzy linear map $\tilde{f}: \chi_{M} \longrightarrow$ $\eta_{N}, \eta_{I m f}$ is called the image of $\tilde{f}$ denoted it by $\eta_{\operatorname{Im} \tilde{f}}$. Further, $\chi_{M_{0}}$, where $M_{0}=\{m \in M \mid \eta(f(m))=1\}$ is called the Kernel of $\tilde{f}$ denoted it by $\chi_{K e r \tilde{f}}$.

Theorem 2.6 ([5]). Let $\tilde{f}: \chi_{M} \longrightarrow \eta_{N}$ be a fuzzy linear map, then $\chi_{K e r \tilde{f}}$ is a fuzzy subspace of $\chi_{M}$ and $\eta_{I m \tilde{f}}$ is a fuzzy subspace of $\eta_{N}$. 
Remark 2.7. For any fuzzy linear map $\tilde{f}: \chi_{M} \longrightarrow \eta_{N}$,

$$
\chi_{K e r f} \leq \chi_{\text {Ker } \tilde{f}} \text { and } \eta_{\text {Imf }}=\eta_{\text {Im } \tilde{f}}
$$

Definition 2.8 ([5]). A fuzzy linear map $\tilde{f}: \chi_{M} \longrightarrow \eta_{N}$ is called epic (or monic) iff $f: M \longrightarrow N$ is epic (or monic)

Definition 2.9 ([5]). A fuzzy linear map $\tilde{f}: \chi_{M} \longrightarrow \eta_{N}$ is called a quasi-monic iff $\chi_{K e r \tilde{f}}=\chi_{M^{\prime}}$, where $M^{\prime}=\{m \in$ $M \mid \chi(m)=1\}$.

Remark 2.10. Obviously, when $\chi_{K e r \tilde{f}}=\{0\}$, quasimonic is just ordinary monic.

Definition 2.11 ([5]). Two fuzzy maps

$$
\chi_{M} \stackrel{\tilde{f}}{\rightarrow} \eta_{N} \stackrel{\tilde{g}}{\rightarrow} \rho_{V}
$$

are exact at $\eta_{N}$ iff $\eta_{I m \tilde{f}}=\eta_{K e r \tilde{g}}$

Remark 2.12. By the induction, from Definition 2.9, we can define an exact sequence of fuzzy linear maps.

Theorem 2.13 ([5]). A fuzzy $R$-module $\chi_{M}$ is called a fuzzy singular $R$-module iff $\chi(m)=1$ for all $m \in M$, denoted it by 1 .

Theorem 2.14 ([5]). An exact sequence

$$
1 \stackrel{\tilde{i}}{\rightarrow} \chi_{M} \stackrel{\tilde{f}}{\rightarrow} \eta_{N} \stackrel{\tilde{g}}{\rightarrow} \rho_{V} \stackrel{\tilde{j}}{\rightarrow} 1
$$

where the two 1's are the appropriate singular fuzzy $R$ modules and $\tilde{i}, \tilde{j}$ are the fuzzy identity map and an epic map, respectively, is called a short exact sequence of fuzzy linear maps.

Theorem 2.15 ([5]). Given a short exact sequence of fuzzy linear maps,

$$
1 \stackrel{\tilde{i}}{\rightarrow} \chi_{M} \stackrel{\tilde{f}}{\rightarrow} \eta_{N} \stackrel{\tilde{g}}{\rightarrow} \rho_{V} \stackrel{\tilde{j}}{\rightarrow} 1
$$

(1) $\operatorname{Im} \tilde{i}=\operatorname{Ker} \tilde{f}=\chi_{M^{\prime}}$.

(2) $\operatorname{Im} \tilde{f}=\operatorname{Ker} \tilde{g} \geq \eta_{N^{\prime}}$.

(3) $\tilde{g}$ is epic,

where $M^{\prime}=\{m \in M \mid \chi(m)=1\}$ and $N^{\prime}=\{n \in N \mid$ $\eta(n)=1\}$.

Proposition 2.16 ([3]). For any fuzzy linear map $\tilde{f}$ : $\chi_{M} \longrightarrow \eta_{N}$,

$$
\chi_{M^{\prime}} \subseteq \chi_{K e r \tilde{f}},
$$

where $M^{\prime}=\{m \in M \mid \chi(m)=1\}$.

\section{Connections with two exact sequences}

In this section, we investigate some situations in connection with exact sequences of fuzzy linear maps of fuzzy $R$-modules.

Kim [6] investigated the other situation (Theorem 3.2) in connection with two exact sequences of fuzzy linear maps of fuzzy R-modules.

Theorem 3.1. Consider the commutative diagram of two exact sequences of fuzzy linear maps, where $\tilde{j}$ is the identity map.

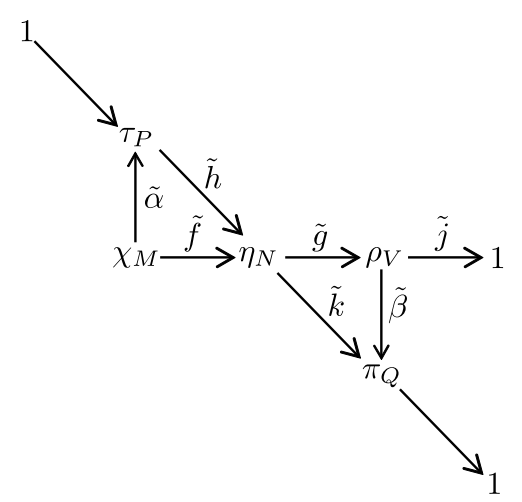

Then

(1) $\tilde{\alpha}$ is quasi-monic.

(2) $\tilde{\beta}$ is epic.

(3) If $\tilde{\alpha}$ is epic, then $\tilde{\beta}$ is quasi-monic.

Proof. (1) To prove that $\tilde{\alpha}$ is quasi-monic, we must show that

$$
\chi_{K e r \tilde{\alpha}} \subseteq \chi_{M^{\prime}},
$$

where $M^{\prime}=\{m \in M \mid \chi(m)=1\}$ by Proposition 2.16 . Let $x \in \chi_{\text {Ker } \tilde{\alpha}}$. Then $\tau(\alpha(x))=1$. Thus $\alpha(x) \in \tau_{P^{\prime}}$, where $P^{\prime}=\{x \in P \mid \tau(x)=1\}$. Since $h$ is a fuzzy $R$-map,

$$
\eta(h \alpha(x)) \geq \tau(\alpha(x)) .
$$

So $\eta((h \alpha(x))=1$. Thus $\alpha(x) \in \operatorname{Ker} \tilde{h}$. Since Ker $\tilde{h}=$ $\tau_{P^{\prime}}, \tau \alpha(x)=1$. Thus $x \in K$ er $\tilde{\alpha}$. Hence $\tilde{\alpha}$ is quasi-monic.

(2) Let $y \in Q$. Since $\tilde{k}$ is epic, there exists $x \in N$ such that $k(x)=y$ and so $g(x) \in V$. Thus, by the commutativity of the diagram $\beta(g(x))=k(x)=y$. Hence $\tilde{\beta}$ is epic.

(3) To prove that $\tilde{\beta}$ is quasi-monic, we must show that

$$
\rho_{K e r \tilde{\beta}} \subseteq \rho_{V^{\prime}},
$$

where $V^{\prime}=\{v \in V \mid \rho(v)=1\}$ by Proposition 2.16. Let $x \in \rho_{\text {Ker } \tilde{\beta}}$. Then $\pi(\beta(x))=1$ and so $\beta(x) \in \pi_{Q^{\prime}}$, where $Q^{\prime}=\{x \in Q \mid \pi(x)=1\}$. Since $\tilde{g}$ is epic, there exists 
$y \in N$ such that $g(y)=x$. Thus, by the commutativity of the diagram

$$
k(y)=\beta g(y)=\beta(x) \in \pi_{Q^{\prime}} .
$$

Thus $\pi k(y)=1$ and so $y \in \eta_{K e r \tilde{k}}$. Since $\eta_{K e r \tilde{k}}=\eta_{I m \tilde{h}}$, there exists $z \in P$ such that $h(z)=y$. Since $\tilde{\alpha}$ is epic, there exists $u \in M$ such that $\alpha(u)=z$. So, by the commutativity of the diagram

$$
f(u)=h \alpha(u)=h(z)=y
$$

and so $y \in \operatorname{Im} \tilde{f}$. Since $\operatorname{Im} \tilde{f}=\operatorname{Ker} \tilde{g}$, we have $\rho g(y)=$ $\rho(x)=1$. Hence $x \in \rho_{V^{\prime}}$. Therefore $\beta$ is quasi-monic.

Theorem 3.2 ([6]). Let the following diagram of fuzzy linear maps of fuzzy $R$-modules be commutative and let the two rows be exact.

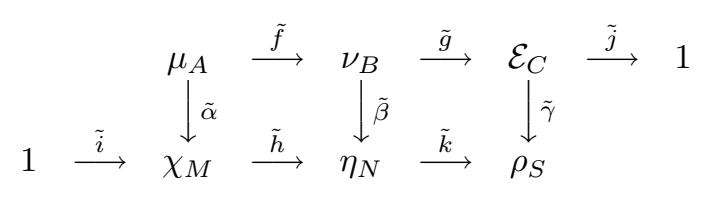

Then

(1) If $\tilde{\alpha}$ and $\tilde{\gamma}$ are epic, then $\tilde{\beta}$ is epic.

(2) If $\tilde{\alpha}$ and $\tilde{\gamma}$ are quasi-monic, then $\tilde{\beta}$ is quasi-monic.

Theorem 3.3. Let the following diagram of fuzzy linear maps of fuzzy $R$-modules be commutative and let the two rows be exact.

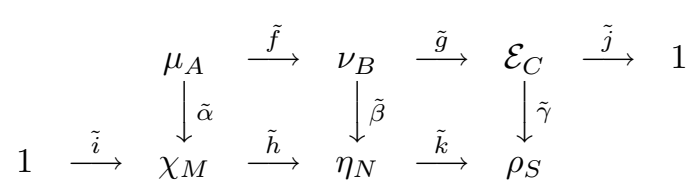

Then

(1) If $\tilde{\beta}$ and $\tilde{f}$ are quasi-monic, then $\tilde{\alpha}$ is quasi-monic.

(2) If $\tilde{\beta}$ and $\tilde{k}$ are quasi-monic, then $\tilde{\gamma}$ is quasi-monic.

Proof. (1) Suppose that $\tilde{\beta}$ and $\tilde{f}$ are quasi-monic. To prove that $\tilde{\alpha}$ is quasi-monic, we must show that

$$
\mu_{K e r \tilde{\alpha}} \subseteq \mu_{A^{\prime}},
$$

where $A^{\prime}=\{a \in A \mid \mu(a)=1\}$ by Proposition 2.16. Let $x \in \mu_{K e r \tilde{\alpha}}$. Then $\chi \alpha(x)=1$. Since $\tilde{h}$ is a fuzzy linear map, $\eta h \alpha(x) \geq \chi \alpha(x)=1$. So $\eta h \alpha(x)=1$. Since $h \alpha(x)=\beta f(x)$ by the commutativity of the diagram,

$$
\eta \beta f(x)=1
$$

Thus $f(x) \in \nu_{K e r \tilde{\beta}}$. Since $\tilde{\beta}$ is quasi-monic, $f(x) \in \nu_{B^{\prime}}$, where $B^{\prime}=\{b \in B \mid \nu(b)=1\}$. Thus $\nu f(x)=1$ and thus $x \in \mu_{K e r \tilde{f}}$. Since $\tilde{f}$ is quasi-monic, we have $x \in \mu_{A^{\prime}}$. Hence $\tilde{\alpha}$ is quasi-monic.

(2) Suppose that $\tilde{\beta}$ and $\tilde{k}$ are quasi-monic. To prove that $\tilde{\gamma}$ is quasi-monic, we must show that

$$
\mathcal{E}_{K e r \tilde{\gamma}} \subseteq \mathcal{E}_{C^{\prime}},
$$

where $C^{\prime}=\{c \in C \mid \mathcal{E}(c)=1\}$ by Proposition 2.16. Let $x \in \mathcal{E}_{\text {Ker } \tilde{\gamma}}$. Then $\rho \gamma(x)=1$. Since $\tilde{g}$ is epic, there exists $y \in B$ such that $g(y)=x$. Thus by commutativity of the diagram,

$$
\rho k \beta(y)=\rho \gamma g(y)=\rho \gamma(x)=1 .
$$

So $\beta(y) \in \eta_{\text {Ker } \tilde{k}}$. Since $\tilde{k}$ is quasi-monic, $\beta(y) \in \eta_{N^{\prime}}$, where $N^{\prime}=\{n \in N\lfloor\eta(n)=1\}$. So $\eta \beta(y)=1$ and so $y \in \nu_{\operatorname{Ker} \tilde{\beta}}$. Since $\tilde{\beta}$ is quasi-monic, $y \in \nu_{B^{\prime}}$. Thus $\nu(y)=1$. Since $g$ is a fuzzy $R$-map,

$$
\mathcal{E}(x)=\mathcal{E} g(y) \geq \nu(y)
$$

Thus $\mathcal{E}(x)=1$ and thus $x \in \mathcal{E}_{C^{\prime}}$. Hence $\gamma$ is quasi-monic.

Theorem 3.4. If, in the following diagram of fuzzy linea mas of fuzzy $R$-modules,

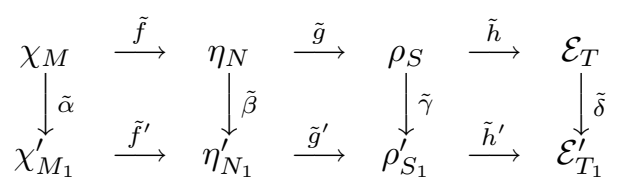

the two rows are exact, the three squares are commutative, $\tilde{\alpha}$ is epic, and $\tilde{\delta}$ is quasi-monic, then

(1) $\eta_{\operatorname{Im} \tilde{\beta}}^{\prime}=\tilde{g}^{\prime-1}\left(\rho_{\operatorname{Im} \tilde{\gamma}}^{\prime}\right)$.

(2) If $g \circ f=0$, then $\rho_{K e r \tilde{\gamma}}=\tilde{g}\left(\eta_{K e r \tilde{\beta}}\right)$.

(3) If $\tilde{\gamma}$ is epic, then so is $\tilde{\beta}$.

(4) If $\tilde{\beta}$ is quasi-monic and $g \circ f=0$, then $\tilde{\gamma}$ is quasimonic.

Proof. (1) Let $y \in \eta_{\text {Im } \tilde{\beta}}^{\prime}$. Then there exists $x \in N$ such that $\beta(x)=y$. By the commutativity of diagram,

$$
g^{\prime}(y)=g^{\prime} \beta(x)=\gamma g(x) \in \rho_{\text {Im } \tilde{\gamma}}^{\prime} .
$$

Thus $y \in \tilde{g}^{\prime-1}\left(\rho_{\operatorname{Im} \tilde{\gamma}}^{\prime}\right)$. Hence $\eta_{I m \tilde{\beta}}^{\prime} \subseteq \tilde{g}^{\prime-1}\left(\rho_{\text {Im } \tilde{\gamma}}^{\prime}\right)$.

Conversely let $y^{\prime} \in{\tilde{g^{\prime}}}^{-1}\left(\rho_{\text {Im }}^{\prime}\right)$ and let $g^{\prime}\left(y^{\prime}\right)=x^{\prime}$. Then there exists $z \in S$ such that $\gamma(z)=x^{\prime}$, since $x^{\prime}=g^{\prime}\left(y^{\prime}\right) \in \rho_{I m \tilde{\gamma}}^{\prime}$. Also $x^{\prime} \in \rho_{\text {Im } \tilde{g}^{\prime}}^{\prime}$. Since $\rho_{\text {Ker } \tilde{h}^{\prime}}^{\prime}=$ $\rho_{\text {Im } g^{\prime}}^{\prime}$, we have $x^{\prime} \in \rho_{\text {Ker } \tilde{h}^{\prime}}$. Thus

$$
\mathcal{E}^{\prime}\left(h^{\prime}\left(x^{\prime}\right)\right)=1 \text {. }
$$


Since $\delta h(z)=h^{\prime} \gamma(z)=h^{\prime}\left(x^{\prime}\right)$, we have

$$
\mathcal{E}^{\prime}(\delta(h(z)))=\mathcal{E}^{\prime}\left(h^{\prime}\left(x^{\prime}\right)=1 .\right.
$$

Thus $h(z) \in \mathcal{E}_{K e r \tilde{\delta}}$ and thus $\mathcal{E}(h(z))=1$, since $\tilde{\delta}$ is quasimonic. So $z \in \rho_{\text {Ker } \tilde{h}}$. Since $\rho_{\text {Ker } \tilde{h}}=\rho_{\text {Im } \tilde{g}}$, there exists $a \in N$ such that $g(a)=z$. Thus

$$
x^{\prime}=\gamma(z)=\gamma(g(a))=g^{\prime} \beta(a) .
$$

Consider $y^{\prime}-\beta(a)\left(\in N_{1}\right)$. Then we get

$$
g^{\prime}\left(y^{\prime}-\beta(a)\right)=g^{\prime}\left(y^{\prime}\right)-g^{\prime} \beta(a)=x^{\prime}-x^{\prime}=0 .
$$

Thus

$$
y^{\prime}-\beta(a) \in \eta_{K e r g^{\prime}} \subseteq \eta_{K e r \tilde{g}^{\prime}}=\eta_{I m \tilde{f}^{\prime}} .
$$

and thus there exists $b^{\prime} \in M_{1}$ such that $f^{\prime}\left(b^{\prime}\right)=y^{\prime}-\beta(a)$. Since $\tilde{\alpha}$ is epic, there exists $b \in M$ such that $\alpha(b)=b^{\prime}$ and $f(b)+a \in N$. So

$$
\begin{aligned}
\beta(f((b)+a) & =\beta(f(b))+\beta(a) \\
& =f^{\prime} \alpha(b)+\beta(a) \\
& =f^{\prime} \alpha\left(b^{\prime}\right)+\beta(a) \\
& =y^{\prime}-\beta(a)+\beta(a) \\
& =y^{\prime} .
\end{aligned}
$$

So $y^{\prime} \in \eta_{\operatorname{Im} \tilde{\beta}}^{\prime}$. Hence $\tilde{g}^{\prime-1}\left(\rho_{\operatorname{Im} \tilde{\gamma}}^{\prime}\right) \subseteq \eta_{\operatorname{Im} \tilde{\beta}}^{\prime}$. This completes the proof of (1).

(2) Let $c \in \rho_{k e r \tilde{\gamma}}$. Then $\rho^{\prime} \gamma(c)=1$. Since $\tilde{h}^{\prime}$ is a fuzzy $R$-map,

$$
\mathcal{E}^{\prime} h^{\prime} \gamma(c) \geq \rho^{\prime} \gamma(c)
$$

So $\mathcal{E}^{\prime} h^{\prime} \gamma(c)=1$. By the commutativity of the diagram, $\delta h(c)=h^{\prime} \gamma(c)$. Thus

$$
\mathcal{E}^{\prime} \delta h(c)=\mathcal{E}^{\prime} h^{\prime} \gamma(c)=1
$$

So $h(c) \in \mathcal{E}_{K e r \tilde{\delta}}$. Since $\tilde{\delta}$ is quasi-monic, $\mathcal{E} h(c)=1$ and so $c \in \rho_{K e r \tilde{h}}$. Since $\rho_{K e r \tilde{h}}=\rho_{I m \tilde{g}}, c \in \rho_{I m \tilde{g}}$. Thus there exists $b \in N$ such that $g(b)=c$. Let $b^{\prime}=\beta(b)\left(\in N_{1}\right)$. Then

$$
g^{\prime}\left(b^{\prime}\right)=g^{\prime} \beta(b)=\gamma g(b)=\gamma(c)
$$

by the commutativity of the diagram and so

$$
\rho^{\prime} g^{\prime}\left(b^{\prime}\right)=\rho^{\prime} \gamma(c)=1 \text {. }
$$

Thus $b^{\prime} \in \eta_{\text {Kerg }}^{\prime}$. Since $\eta_{\text {Kerg }}^{\prime} \tilde{g}^{\prime}=\eta_{I m \tilde{f}^{\prime}}^{\prime}$ by the commutativity of the diagram, there exists $a^{\prime} \in M_{1}$ such that $f^{\prime}\left(a^{\prime}\right)=b^{\prime}$. Since $\alpha$ is epic, there exists $a \in M$ such that $\alpha(a)=a^{\prime}$. Consider $b-f(a)(\in N)$. Then we have

$$
\begin{aligned}
\beta(b-f(a)) & =\beta(b)-\beta(f(a)) \\
& =\beta(b)-f^{\prime} \alpha(a) \\
& =b^{\prime}-b^{\prime} \\
& =0 .
\end{aligned}
$$

Hence

$$
b-f(a) \in \eta_{\text {Ker } \beta} \subseteq \eta_{\text {Ker } \tilde{\beta}} .
$$

and since $g \circ f=0$, we obtain

$$
\begin{aligned}
g(b-f(a)) & =g(b)-g f(a) \\
& =c .
\end{aligned}
$$

Thus $c \in \tilde{g}\left(\eta_{k e r \tilde{\beta}}\right)$ and thus $\rho_{k e r \tilde{\gamma}} \subseteq \tilde{g}\left(\eta_{k e r \tilde{\beta}}\right)$.

Conversely let $c \in \tilde{g}\left(\eta_{\text {ker } \tilde{\beta}}\right)$. Then there exists $b \in \eta_{\text {ker } \tilde{\beta}}$ such that $g(b)=c$. Thus

$$
\eta^{\prime} \beta(b)=1 \text {. }
$$

and thus $\gamma(c)=\gamma g(b)=g^{\prime} \beta(b)$. Since $g^{\prime}$ is a fuzzy $R$ map,

$$
\rho^{\prime} \gamma(c)=\rho^{\prime} g^{\prime} \beta(b) \geq \eta^{\prime} \beta(b)=1 .
$$

Thus $\rho^{\prime} \gamma(c)=1$. and thus $c \in \rho_{\text {Ker } \tilde{\gamma}}$. Hence $\tilde{g}\left(\eta_{k e r \tilde{\beta}}\right) \subseteq$ $\rho_{\text {ker } \tilde{\gamma}}$. This completes the proof of (2).

(3) Suppose that $\tilde{\gamma}$ is epic. Then $\operatorname{Im} \tilde{\gamma}=S_{1}$ and then

$$
\begin{aligned}
\eta_{\text {Im } \tilde{\beta}}^{\prime} & ={\tilde{g^{\prime}}}^{-1}\left(\rho_{\text {Im } \tilde{\gamma}}^{\prime}\right) \\
& =\tilde{g}^{\prime-1}\left(S_{1}\right) \\
& =N_{1} .
\end{aligned}
$$

Hence $\tilde{\beta}$ is epic.

(4) Suppose that $\tilde{\beta}$ is quasi-monic. Then

$$
\eta_{\text {Ker } \tilde{\beta}}=\eta_{N^{\prime}}
$$

where $N^{\prime}=\{x \in N \mid \eta(x)=1\}$. To prove that $\tilde{\gamma}$ is quasi-monic, we must show that

$$
\rho_{K e r \tilde{\gamma}}=\rho_{S^{\prime}},
$$

where $S^{\prime}=\{x \in S \mid \rho(x)=1\}$. Let $x \in \rho_{K e r \tilde{\gamma}}$. Then by (2), there exists $y \in \eta_{K \operatorname{Ker} \tilde{\beta}}$ such that $g(y)=x$. Since $\tilde{\beta}$ is quasi-monic, $y \in \eta_{N^{\prime}}$ and so $\eta(y)=1$. Since $\tilde{g}$ is a fuzzy $R$-map,

$$
\rho(x)=\rho g(y) \geq \eta(y)=1 .
$$

Thus $\rho(x)=1$ and thus $x \in \rho_{S^{\prime}}$.

Conversely let $x \in \rho_{S^{\prime}}$. Then $\rho(x)=1$. Since $\tilde{\gamma}$ is a fuzzy $R$-map,

$$
\rho^{\prime} \gamma(x) \geq \rho(x)=1 \text {. }
$$

Thus $\rho^{\prime} \gamma(x)=1$ and thus $x \in \rho_{K e r \tilde{\gamma}}$. Hence $\tilde{\gamma}$ is quasimonic.

Corollary 3.5. If, in the following diagram of fuzzy linea maps of fuzzy $R$-modules,

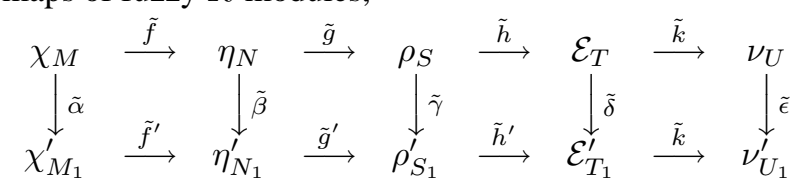

the two rows are exact, the four squares are commutative, the homomorphisms $\tilde{\alpha}, \tilde{\beta}, \tilde{\delta}, \tilde{\epsilon}$ are fuzzy weak isomorphisms, and $g \circ f=0$, then the middle homomorphism $\tilde{\gamma}$ is a fuzzy weak isomorphism. 
Proof. By Theorem 3.4, $\tilde{\gamma}$ is epic. Also by definition of a fuzzy $R$-map, $\rho^{\prime} \gamma(c) \geq \rho(c)$ for all $c \in S$. To prove that $\tilde{\gamma}$ is a fuzzy weak isomorphism, we must show that $\gamma$ is monic. Let $x \in \operatorname{Ker} \gamma$. Then $\gamma(x)=0$. So by the commutativity of the diagram,

$$
\delta h(x)=h^{\prime} \gamma(x)=h^{\prime}(0)=0 .
$$

Thus $h(x) \in K e r \delta$. Since $\delta$ is monic, $h(x)=0$. So

$$
x \in \rho_{K e r h} \subseteq \rho_{K e r \tilde{h}} .
$$

Since $\rho_{\text {Ker } \tilde{h}}=\rho_{\text {Im } \tilde{g}}$, there exists $n \in N$ such that $g(n)=$ $x$. Thus by the commutativity of the diagram,

$$
g^{\prime} \beta(n)=\gamma g(n)=\gamma(x)=0
$$

So

$$
\beta(b) \in \eta_{\text {Kerg }}^{\prime} \subseteq \eta_{K e r \tilde{g}^{\prime}}^{\prime} .
$$

Since $\eta_{\text {Ker } \tilde{g}^{\prime}}^{\prime}=\eta_{I m \tilde{f}^{\prime}}^{\prime}$, there exists $m^{\prime} \in M_{1}$ such that $f^{\prime}\left(m^{\prime}\right)=\beta(n)$. Since $\alpha$ is epic, there exists $m \in M$ such that $\alpha(m)=m^{\prime}$ Thus

$$
\beta f(m)=f^{\prime} \alpha(m)=\beta(n) .
$$

So

$$
\beta(f(m)-n)=0 .
$$

Thus $f(m)-n \in \nu_{K e r \beta} \subseteq \nu_{K e r} \tilde{\beta}$. Since $\beta$ is monic, $f(m)=n$. Thus since $g \circ f=0$,

$$
x=g(n)=g(f(m)=0 .
$$

Hence $\gamma$ is monic. Therefore $\gamma$ is a fuzzy weak isomorphism.

Theorem 3.6. Consider the following commutative square of fuzzy $R$-maps of $R$-modules:

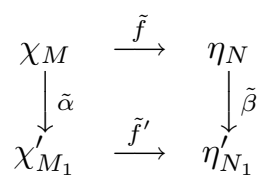

Then $\tilde{f}$ carries $\chi_{K e r \tilde{\alpha}}$ into $\eta_{K e r \tilde{\beta}}$. (In this case, $\tilde{f}$ is denoted by $\tilde{f}^{*}$.)

Proof. Let $x \in \chi_{K e r \tilde{\alpha}}$. Then $\chi^{\prime} \alpha(x)=1$. Thus

$$
\begin{aligned}
\eta^{\prime} \beta f(x) & =\eta^{\prime} f^{\prime} \alpha(x) \\
& \geq \chi^{\prime} \alpha(x) \\
& =1 .
\end{aligned}
$$

So $\eta^{\prime} \beta f(x)=1$. Hence $f(x) \in \eta_{K e r \tilde{\beta}}$. This completes the proof.

From Theorem 3.6, we obtain the following theorem.
Theorem 3.7. . Consider the following diagram of fuzzy $R$-maps of $R$-modules :

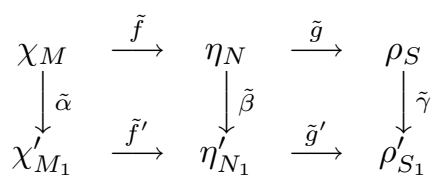

where the rows are exact and the squares are commutative. If $\tilde{f}^{\prime}: \chi_{M_{1}}^{\prime} \longrightarrow \eta_{N_{1}}^{\prime}$ is quasi-monic, then the sequence of fuzzy $R$-maps,

$$
\chi_{K e r \tilde{\alpha}} \stackrel{\tilde{f}^{*}}{\rightarrow} \eta_{K e r \tilde{\beta}} \stackrel{\tilde{g}^{*}}{\rightarrow} \rho_{K e r \tilde{\gamma}}
$$

is exact at $\eta_{K e r \tilde{\beta}}$.

Proof. We must show that $\eta_{I m \tilde{f}^{*}}=\eta_{\text {Ker } \tilde{g}^{*}}$. Let $a \in$ $\eta_{\operatorname{Im} \tilde{f}^{*}}$. There there exists $b \in \operatorname{Ker} \tilde{\alpha}$ such that $f^{*}(b)=a$. Since $a=f^{*}(b)=f(b)$,

$$
a \in \eta_{I m \tilde{f}}
$$

Since $\eta_{I m \tilde{f}}=\eta_{K e r \tilde{g}}$,

$$
\rho g(a)=1 .
$$

So $\rho g^{*}(a)=1$ and so $a \in \eta_{\text {Ker } \tilde{g}^{*}}$. Hence

$$
\eta_{I m \tilde{f^{*}}} \subseteq \eta_{K e r \tilde{g}^{*}}
$$

Conversely let $a \in \eta_{K e r g^{*}}\left(a \in \eta_{K e r \tilde{\beta}}\right)$. Then $\rho g^{*}(a)=$ 1 and so $\rho g(a)=1$. Thus

$$
a \in \eta_{K e r \tilde{g}} .
$$

Since $\eta_{K e r \tilde{g}}=\eta_{\operatorname{Im} \tilde{f}}$, there exists $c \in A$ such that $f(c)=$ $a$. Since $a \in \eta_{K e r \tilde{\beta}}$,

$$
\eta^{\prime} \beta(a)=1
$$

Thus

$$
\begin{aligned}
\eta^{\prime} f^{\prime} \alpha(c) & =\eta^{\prime} \beta f(c) \\
& =\eta^{\prime} \beta(a) \\
& =1
\end{aligned}
$$

So

$$
\alpha(c) \in \chi_{K e r \tilde{f}^{\prime}}^{\prime}
$$

Since $\tilde{f}^{\prime}$ is quasi-monic,

$$
\chi^{\prime}(\alpha(c))=1 \text {. }
$$

Thus $c \in \chi_{\text {Ker } \tilde{\alpha}}$ and thus $f^{*}(c)=f(c)=a$. So $a \in$ $\eta_{\text {Im } \tilde{f}^{*}}$. Hence $\eta_{K e r \tilde{g}^{*}} \subseteq \eta_{\text {Im } \tilde{f}^{*}}$. This completes the proof.

\section{Acknowledgements}

I express my thanks to the referees for their valuable comments and suggestions. 


\section{References}

[1] C. V. Negoita and D. A. Ralescu, "Applications of Fuzzy Sets to System Analysis", Birkhäuser, Basel, Chap. 1, pp.18-24, 1975.

[2] A. K. Katsaras and D. B. Liu, "Fuzzy Vector Spaces and Fuzzy Topological Vector Spaces", J. of Math. Anal. Appl., 58 , pp. 135-146, 1977.

[3] R. Lowen, “Convex Fuzzy Sets”, Fuzzy Sets and Systems, vol. 3, no. 3, pp. 291-310, 1980.

[4] F. Z. Pan, "Fuzzy Finitely Gernerated Modules", Fuzzy Sets and Systems, vol. 21, no. 1, pp. 105-113, 1987.

[5] F. Z. Pan, "Exact Sequences of Fuzzy Linear Maps", Fuzzy Sets and Systems, vol. 27, no. 3, pp. 317-325, 1988.
[6] C. B. Kim, "The Fuzzy Linear Maps", J. of Fuzzy Logic and Intelligent Systems, vol. 15, no. 4, pp. 511-513, 2005.

[7] S. T. Hu, Introduction to Homological Algebra, Holden-day, Inc. San Francisco, 1968.

[8] N. G. Northcott, An Introduction to Homological Algebra, Cambridge Univ. press, London, 1960.

[9] J. J. Rotman, An Introduction to Homological Algebra, Academic press, New York, 1972.

\section{Chang Bum Kim}

Professor of Kookmin University

Research Area : Fuzzy Mathematics, Fuzzy Algebra

E-mail : cbkim@kookmin.ac.kr 